Pacific Journal of Mathematics

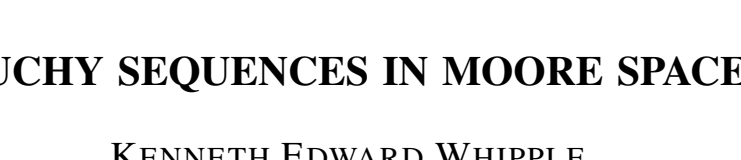




\title{
CAUCHY SEQUENCES IN MOORE SPACES
}

\author{
KenNeth E. WhipPle
}

\begin{abstract}
In this paper the concept of a Cauchy sequence is extended to Moore spaces. This concept is then used to characterize those Moore spaces which are completable (i.e., topologically equivalent to a subspace of a complete Moore space). The definition for Cauchy sequence given here is shown to be a generalization of the usual definition for Cauchy sequence in a metric space. Also considered are certain questions concerning completability of a Moore space having a dense completable subspace.
\end{abstract}

It is well known that every metric space is a subspace of a complete metric space. In [6] Mary Ellen Rudin proved the existence of Moore spaces that are not subspaces of complete Moore spaces. In [1] O. H. Alzoobaee gave a sufficient condition that a Moore space be a subspace of a complete Moore space, but it was not stated and it appears to be unknown whether this condition is necessary. The results of the present paper were obtained independently of Alzoobaee's paper.

Let $S$ be a topological space and let $G$ be a monotonically decreasing sequence of open coverings of $S$. The statement that $G$ is a development for $S$ means that if $D$ is an open set containing the point $x$, then there exists a positive integer $n$ such that if $R \in G_{n}$ $x \in R$ then $\bar{R} \subset D$. A topological $\left(T_{1}\right)$ space $S$ having a development is called a Moore space. The statement that $G$ is a strong development for $S$ means that if $D$ is an open set containing the point $x$, then there exists a positive integer $n$ such that if $R \in G_{n}$ and $x \in \bar{R}$ then $\bar{R} \subset D$.

If $G$ is a development for the Moore space $S$ and if $H$ is a monotonically decreasing sequence of open coverings of $S$ such that $H_{n}$ is a refinement of $G_{n}$ for each $n$, then $H$ is said to be a refinement of $G$. A refinement of a development is a development, and a refinement of a strong development is a strong development. Furthermore, every development has a refinement that is a strong development.

The statement that a development $G$ for $S$ is complete means that if $T$ is a monotonically decreasing sequence of closed point sets such that $T_{n}$ is contained in an element of $G_{n}$ for each $n$, then there is a point $x$ such that $x \in T_{n}$ for each $n$. A Moore space is said to be complete if it has a complete development. In [6, Th. 3, p. 322], M. E. Rudin proved that a topological $\left(T_{1}\right)$ space satisfies R. L. Moore's Axiom 1 [3] if and only if it has a complete development as defined 
here. Every refinement of a complete development is a complete development.

Every metric space is a Moore space, but the converse is not true. In [4], R. L. Moore gave a necessary and sufficient condition for a Moore space to be metrizable. This condition is restated in Section 3 of this paper. In [5], J. H. Roberts proved that a metrizable space $S$ is a complete Moore space if and only if $S$ is a complete metric space with respect to some metric $d$ for $S$.

Let $M$ be a subspace of the Moore space $S$, let $G$ be a development for $S$, and let $H$ be the sequence such that $H_{n}=\left\{R \cdot M: R \in G_{n}\right\}$. Then $H$ is a development for $M$ and is called the development for $M$ relative to $G$. If $G$ is a strong development for $S$, then $H$ is a strong development for $M$.

2. The Cauchy-sequential separation property. A point sequence $p$ is said to be a Cauchy sequence with respect to a strong development $G$ (or simply Cauchy sequence $(G)$ ) if, for each positive integer $n$, some element of $G_{n}$ contains a final segment of $p$.

If $G$ is a strong development, then every convergent sequence is a Cauchy sequence with respect to $G$. Furthermore, if a Cauchy sequence with respect to $G$ has a convergent subsequence, then the sequence converges. Using these facts, the following theorem can be verified.

THEOREM 1. A strong development $G$ is complete if and only if every Cauchy sequence with respect to $G$ converges.

Let $G$ be a strong development for $S$, and let $D$ be an open set in $S$. Then $p$ is said to be an interior sequence with respect to $G$ of $D$ (or simply interior sequence $(G)$ of $D$ ) if $p$ is a Cauchy sequence with respect to $G$ and for some positive integer $n$ every element of $G_{n}$ that contains a final segment of $p$ is a subset of $D$. A development $G$ is said to have the Cauchy-sequential separation property if $G$ is a strong development having the property that if $p$ is an interior sequence of an open set $D$, then for each positive integer $n$ there is an element $R$ of $G_{n}$ such that $p$ is an interior sequence of $R$ and every Cauchy sequence having a final segment in $R$ is an interior sequence of $D$. (The phrase "with respect to $G$ " is omitted whenever there has been only one development mentioned.)

It follows that if $p$ is a point sequence converging to a point $x$ and if $D$ is an open set, then $x \in D$ if and only if $p$ is an interior sequence of $D$. Furthermore, suppose $H$ is a refinement of a strong development $G, p$ is a Cauchy sequence $(H)$, and $D$ is an open set. 
Then $p$ is a Cauchy sequence $(G)$, and if $p$ is an interior sequence $(G)$ of $D$, then $p$ is an interior sequence $(H)$ of $D$.

THEOREM 2. Suppose $M$ is a subset of the Moore space $S, G$ is a strong development for $S, H$ is the development for $M$ relative to $G$, and $p$ is a sequence of points in $M$. Then (1) $p$ is a Cauchy sequence $(G)$ if and only if $p$ is a Cauchy sequence $(H)$; (2) if $p$ is an interior sequence $(G)$ of an open set $D$ in $S$, then $p$ is an interior sequence $(H)$ of $D \cdot M$; and (3) if $p$ is an interior sequence (H) of an open set $R$ in $M$ and if $R^{\prime}$ is the maximal open set in $S$ such that $R^{\prime} \cdot M=R$, then $p$ is an interior sequence $(G)$ of $R^{\prime}$.

THEOREM 3. If $M$ is a subspace of a complete Moore space $S$ and $H$ is a development for $M$, then there exists a refinement $K$ of $H$ having the Cauchy-sequential separation property.

Proof. Let $G$ be a strong, complete development for $S$, let $E$ be the development for $M$ relative to $G$, and let $F$ be a common refinement of $H$ and $E$. Then $F$ is a strong development for $M$. Let $K$ be the sequence such that $K_{1}=F_{1}$ and such that for each positive integer $n, K_{n+1}$ consists of those elements $R$ of $F_{n+1}$ for which there are an open set $U$ in $S$ and an element $D$ of $K_{n}$ such that $\bar{R} \subset U$ and $U \cdot M \subset D$ (where $\bar{R}$ denotes the closure of $R$ in $S$ ). Then $K$ is a refinement of $F$.

Suppose that $p$ is an interior sequence $(K)$ of an open set $T$ in $M$ and $n$ is a positive integer. Then $p$ is a Cauchy sequence $(G)$; hence $p$ converges to a point $x$ in $S$. There exists an integer $m>n$ such that each element of $K_{m}$ containing a final segment of $p$ is a subset of $T$. Let $R$ be an element of $K_{m+2}$ that contains a final segment of $p$. There exist an open set $U_{1}$ in $S$ and an element $R_{1}$ of $K_{m+1}$ such that $\bar{R} \subset U_{1}$ and $U_{1} \cdot M \subset R_{1}$. Then $p$ is an interior sequence $(K)$ of $R_{1}$.

Suppose that $q$ is a Cauchy sequence $(K)$ having a final segment in $R_{1}$. Then $q$ converges to a point $y$ in $S$. There exists an open set $U_{2}$ in $S$ and an element $R_{2}$ of $K_{m}$ such that $\bar{R}_{1} \subset U_{2}$ and $U_{2} \cdot M \subset R_{2}$. Then $y \in U_{2}$; hence $q$ is an interior sequence $(K)$ of $R_{2}$; and hence $q$ is an interior sequence $(K)$ of $T$.

Suppose that $G$ is strong development for a Moore space $S$. The statement that the Cauchy sequences $p$ and $q$ are equivalent (denoted $p \sim q$ ) means that $p$ is an interior sequence of an open set $D$ if and only if $q$ is an interior sequence of $D$. This relation is an equivalence relation. Furthermore, if $p$ and $q$ are Cauchy sequences such that for each positive integer $n$ some element of $G_{n}$ has both $p$ and $q$ as interior sequences, then $p \sim q$. 
THEOREM 4. If $S$ is a Moore space having a development $G$ with the Cauchy-sequential separation property, then there exists a complete Moore space $S^{\prime}$ such that $S$ is topologically equivalent to a dense subset of $S^{\prime}$. Such a space $S^{\prime}$ is called a completion of $S$, and $S$ is said to be completable.

Proof. For each Cauchy sequence $p$, let $p^{\prime}$ denote the collection of all Cauchy sequences that are equivalent to $p$. For each point $x$ in $S$, let $\dot{x}$ denote the sequence each term of which is $x$. Let $S^{\prime}=$ $\left\{p^{\prime}: p\right.$ is a Cauchy sequence $\left.(G)\right\}$, and for each open set $D$ in $S$, let $D^{\prime}=\left\{p^{\prime}: p\right.$ is an interior sequence of $\left.D\right\}$. The collection $B^{\prime}=\left\{D^{\prime}: D\right.$ is an open set in $S\}$ forms a $T_{1}$ basis for $S^{\prime}$.

If $D$ is an open set and $p$ is a Cauchy sequence, then $p^{\prime} \in \bar{D}^{\prime}$ if and only if there exists a Cauchy sequence $q$ such that $p \sim q$ and $D$ contains a final segment of $q$. To establish this, suppose $p \sim q$ and $D$ contains a final segment of $q$. If $R^{\prime}$ is an element of $B^{\prime}$ containing $p^{\prime}$, then $q_{n} \in R \cdot D$ for some $n$; hence $\dot{q}_{n}^{\prime} \in R^{\prime} \cdot D^{\prime}$. Now, suppose that $p^{\prime} \in \bar{D}^{\prime}$. Let $R_{0}=S$ and for each positive integer $n$, let $R_{n}$ be an element of $G_{n}$ such that $p$ is an interior sequence of $R_{n}$ and every Cauchy sequence having a final segment in $R_{n}$ is an interior sequence of $R_{n-1}$. Let $q$ be a sequence such that $q_{n} \in R_{n} \cdot D$ for each $n$. Then $D$ contains a final segment of $q$, and $p \sim q$.

Hence, it follows that the sequence $G^{\prime}$ such that $G_{n}^{\prime}=\left\{R^{\prime}: R \in G_{n}\right\}$ is a development for $S^{\prime}$. Moreover, $G^{\prime}$ is a complete development, as will now be proved.

Let $T^{\prime}$ be a monotonically decreasing sequence of closed sets in $S^{\prime}$ such that for each $n$ there exists an element $R_{n}^{\prime}$ of $G_{n}^{\prime}$ that contains $T_{n}^{\prime}$. For each positive integer $n$ let $p(n)^{\prime} \in T_{n}^{\prime}$. Then $p(n)$ is an interior sequence of $R_{i}$ for each positive integer $i \leqq n$. Let $q$ be a sequence such that, for each $n, q_{n}$ is a point of $p(n)$ belonging to $R_{1} \cdot R_{2} \cdot \cdots \cdot R_{n}$. Then $q^{\prime}$ is a sequential limit point of the sequence $\{p(1), p(2), \cdots\}$; thus $q^{\prime} \in T_{i}^{\prime}$ for all $i$.

The transformation $F$ from $S$ into $S^{\prime}$ such that $F(x)=\dot{x}^{\prime}$ is a topological transformation from $S$ onto a dense subset of $S^{\prime}$.

3. Metric Spaces. Suppose $G$ is a development for $S$. The statement that $G$ satisfies Axiom $C$ means that if $x$ is a point of an open set $D$ then there is a positive integer $n$ such that every two link chain of $G_{n}$ that contains $x$ is a subset of $D$. If $G$ satisfies Axion $\mathrm{C}$, then $G$ is strong. In [4] R. L. Moore proved that a topological space is metrizable if and only if it has a development which satisfies Axiom C.

While it is true that every metrizable Moore space is completable and every space having a development satisfying Axiom $\mathrm{C}$ is metrizable, 
it is not true that every Axiom $\mathrm{C}$ development has the Cauchy-sequential separation property.

THEOREM 5. There exists a space $S$ having a development $G$ such that $G$ does not have the Cauchy-sequential separation property, but $G$ does have the property that for each positive integer n, every two link chain of $G_{n+1}$ is contained in an element of $G_{n}$.

Proof. Let $S$ be the subspace of $E^{2}$ consisting of the points $(x, y)$ such that $x>0$ and $y / x$ is a positive integer. Let $G$ be the sequence such that $G_{n}$ consists of all point sets of the following types:

(1) $\{(t, m t): a<t<b\}$ where $m$ is a positive integer, $0<a<b$, and $b-a<2^{-n}$;

(2) $\{(x, y): 0<x<r$, and $y / x \in N\}$ where $0<r<2^{-n}$ and $N$ is a finite set of positive integers.

Then $G$ is a strong development, and every two link chain of $G_{n+1}$ is contained in an element of $G_{n}$. But $G$ does not have the Cauchy-sequential separation property since the Cauchy sequence $p$ such that $p_{n}=\left(2^{-n}, 2^{-n}\right)$ is not an interior sequence of any element of $G_{1}$.

A development $G$ for $S$ is said to have property $U$ if, for each positive integer $m$, there is an integer $n>m$ such that if $D \in G_{n}$ there is an element $V$ of $G_{m}$ such that every element of $G_{n}$ that intersects $D$ is contained in $V$.

THeORem 6. If $G$ is a development for $S$ having property $U$, then $G$ satisfies Axiom $C$ and has the Cauchy-sequential separation property.

Proof. Clearly $G$ satisfies Axiom C. That $G$ has the Cauchysequential separation property follows from the fact that if $U$ and $V$ are open sets such that for some integer $n$ every element of $G_{n}$ that intersects $V$ is a subset of $U$, then every Cauchy sequence having a final segment in $V$ is an interior sequence of $U$.

THEOREM 7. If $d$ is a metric defined on $S$, then the following statements are true.

(1) There exist sequences $u$ and $v$ of positive numbers converging to 0 and a monotonically decreasing sequence $G$ of open coverings of $S$ such that if $R \in G_{n}$ then diam $(R) \leqq u_{n}$ and if $H$ is a point set such that $\operatorname{diam}(H) \leqq v_{n}$ then $H$ is contained in some element of $G_{n}$. 
(2) Every such sequence $G$ is a development for $S$ having property $U$.

Proof of (1). Let $u, v$, and $G$ be the sequences that, for each $n$, $u_{n}=1 / n, v_{n}=1 / 3 n$, and $G_{n}=\left\{R_{r}(y): y \in S\right.$ and $\left.0<r<1 / 2 n\right\}$ where $R_{r}(y)=\{x \in S: d(x, y)<r\}$.

Proof of (2). Clearly, $G$ is a development for $S$. Suppose $m$ is a positive integer. Let $n$ be an integer greater than $m$ such that $u_{n}<v_{m} / 4$. Suppose $R \in G_{n}$. Let $y \in R$ and let $H=\left\{x: d(y, x)<v_{m} / 2\right\}$. Then $\operatorname{diam}(H) \leqq v_{m}$; hence $H$ is contained in some element of $G_{m}$. But every element of $G_{n}$ that intersects $R$ is a subset of $H$. Hence, $G$ has property $U$.

Let $G$ be a sequence as in Theorem 7 . Then a point sequence $p$ is a Cauchy sequence with respect to $G$ if and only if for each positive number $c$ there is a positive integer $n$ such that $d\left(p_{i}, p_{j}\right)<c$ for all integers $i$ and $j$ greater than $n$. Hence if $p$ and $q$ are Cauchy sequences, the real number sequence $r$ such that $r_{n}=d\left(p_{n}, q_{n}\right)$ converges. Let $\hat{d}(p, q)$ denote this limit. Then $p \sim q$ if and only if $\hat{d}(p, q)=0$. If $p$ is an interior sequence of an open set $D$, there exists a positive number $c$ such that every Cauchy sequence $q$ for which $\hat{d}(p, q)<c$ is an interior sequence of $D$. Furthermore, if $p$ is a Cauchy sequence and $c$ is a positive number, there exists an open set $R$ having $p$ as an interior sequence such that if $q$ is an interior sequence of $R$ then $\hat{d}(p, q)<c$. In other words, the usual completion of a metric space is the same as the completion that is obtained by the methods of Theorem 4 whenever $G$ is a development having the properties of Theorem 7 with respect to the given metric.

4. Dense subspaces. Suppose $M$ is a dense subspace of $S, G$ is a strong development for $S$, and $H$ is the development for $M$ relative to $G$. If $G$ has the Cauchy-sequential separation property then so does $H$ (Theorem 8), but the converse is not true (Theorem 9). However, if $H$ has the Cauchy-sequential separation property, there does exist a development $H^{\prime}$ for $S$ having this property (Theorem 10). It is not true, however, that if $M$ is completable (or even complete) then $S$ is completable (Theorem 11).

THEOREM 8. If $M$ is a subspace of the Moore space $S$ and if $G$ is a development for $S$ having the Cauchy-sequential separation property, then the development for $M$ relative to $G$ has the Cauchysequential separation property. 


\section{Proof. This is a direct consequence of Theorem 2 .}

THeOREM 9. There exist a space $S$, a dense subset $M$ of $S$, and a development $G$ for $S$ such that $G$ does not have the Cauchy-sequential separation property, but the development for $M$ relative to $G$ has the Cauchy-sequential separation property.

Proof. Let $S$ be the subspace of $E^{2}$ consisting of the points $(x, y)$ for which $x \geqq 0$ and $y \geqq 0$, and let $G$ be the development such that $G_{n}$ consist of the following types of point sets:

(1) $\left\{(x, y): x \geqq 0, y \geqq 0\right.$, and $\left.(x-a)^{2}+(y-b)^{2}<r^{2}\right\}$ where $a \geqq 0$, $b \geqq 0$, and $0<r<1 / n$;

(2) $\{(x, y): x>t$ and $0<y<1 / t\}+\{(x, 0): m-1 / 4<x<m+$ $1 / 2$ for each integer $m>t+1 / 4\}$ where $t \geqq n$;

(3) $\{(x, y): x>t$ and $0<y<1 / t\}+\{(x, 0): m-1 / 2<x<m+$ $1 / 4$ for each integer $m>t+1 / 2\}$ where $t \geqq n$.

Then $G$ does not have the Cauchy-sequential separation property, since the sequence $p$ such that $p_{n}=(n, 0)$ is a Cauchy sequence $(G)$ but is not an interior sequence of any element of $G_{1}$. However, the set $M=\{(x, y): x>0$ and $y>0\}$ is dense in $S$, and the development for $M$ relative to $G$ has the Cauchy-sequential separation property.

THeOREM 10. Suppose $G$ is a development for a space $S, M$ is $a$ dense subset of $S, H$ is the development for $M$ relative to $G$, and $H$ has the Cauchy-sequential separation property. Then there is a strong development $H^{\prime}$ for $S$ having the Cauchy-sequential separation property. Furthermore, $H$ is the development for $M$ relative to $H^{\prime}$, and $G$ is a refinement of $H^{\prime}$.

Proof. For each open set $D$ in $M$, let $D^{\prime}$ denote the maximal open set in $S$ such that $D^{\prime} \cdot M=D$. Let $H^{\prime}$ be the sequence such that $H_{n}^{\prime}=\left\{R^{\prime}: R \in H_{n}\right\}$. Then $H^{\prime}$ is a strong development for $S$, and $H$ is the development for $M$ relative to $H^{\prime}$.

That $H^{\prime}$ has the Cauchy-sequential separation property follows once it is shown that if $K$ is an open set in $S$ containing a final segment of the Cauchy sequence $p$ with respect to $H^{\prime}$ then there is a Cauchy sequence $q$ (with respect to $H$ and to $H^{\prime}$ ) of points in $M \cdot K$ such that $p \sim q$. To this end, let $R_{n}^{\prime}$ be an element of $H_{n}^{\prime}$ containing a final segment of $p$ for each $n$, and let $q$ be a sequence such that $q_{n} \in$ $M \cdot K \cdot R_{1}^{\prime} \cdot \cdots \cdot R_{n}^{\prime}$. Then each $R_{n}^{\prime}$ contains final segments of $p$ and $q$. If $p$ or $q$ is an interior sequence $\left(H^{\prime}\right)$ of an open set $D$ in $S$, then $D$ contains final segments of both $p$ and $q$.

Suppose $p$ is an interior sequence $\left(H^{\prime}\right)$ of an open set $D$ in $S$. There exists a positive integer $m$ such that every element of $H_{m}^{\prime}$ 
containing a final segment of $p$ is contained in $D$. Now $q$ is an interior sequence $(H)$ of some element $U$ of $H_{m}$; hence $U^{\prime}$ is an element of $H_{m}^{\prime}$ that contains a final segment of $p$; hence $U^{\prime} \subset D$, and $q$ is an interior sequence $\left(H^{\prime}\right)$ of $D$.

Suppose $q$ is an interior sequence $\left(H^{\prime}\right)$ of an open set $E$ in $S$. There exists an open set $U$ in $M$ such that $q$ is an interior sequence $(H)$ of $U$, and $U^{\prime} \subset E$. There is an open set $V$ in $M$ such that $q$ is an interior sequence $(H)$ of $V$ and every Cauchy sequence $(H)$ of points in $M$ having a final segment in $V$ is an interior sequence $(H)$ of $U$. Then $V^{\prime}$ contains a final segment of $p$. Assume that $p$ is not an interior sequence $\left(H^{\prime}\right)$ of $U^{\prime}$. For each positive integer $n$, let $W_{n}^{\prime}$ be an element of $H_{n}^{\prime}$ containing a final segment of $p$ that is not contained in $U^{\prime}$. Let $r$ be a sequence such that

$$
r_{n} \in M \cdot V^{\prime} \cdot W_{1}^{\prime} \cdot W_{2}^{\prime} \cdot \cdots \cdot W_{n}^{\prime} .
$$

Then $r$ is an interior sequence $(H)$ of $U$; hence there is an integer $k$ such that $W_{k} \subset U$. This implies that $W_{k}^{\prime} \subset U^{\prime}$ which is a contradiction. Thus $p$ in an interior sequence of $E$.

In [2], B. Fitzpatrick proved that every completable Moore space contains a dense metrizable subspace. In conversation with this author he raised several questions concerning dense subspaces of noncompletable spaces some of which are settled by the following example due to M. E. Rudin.

THEOREM 11. There exists a noncompletable Moore space S such that (1) $S$ contains a complete metric subspace that is dense in $S$, (2) $S$ is locally metrizable (and therefore, locally completable), and (3) every subspace of $S$ contains a dense metric subspace.

Proof. Let $U=\left\{\sum_{n \in N} 4^{-n}: N\right.$ is a finite set of positive integers $\}$ and let $V=\bar{U}-U$ (with respect to the topology of $E^{1}$ ). Let $M=$ $U \times 0$ and let $F=V \times I(0,1)$. Let $B$ be a maximal collection of number sequences such that (1) if $d \in B$ then $d_{i} \in V \cdot I\left(4^{-i-1}, 4^{-i}\right)$ for each $i$, and (2) if $c$ and $d$ are distinct elements of $B$ then there is a positive integer $n$ such that $c_{i} \neq d_{i}$ for all integers $i \geqq n$.

Let $S$ be the space having point to be an element of $M, F$, or $B$ and having a basis consisting of all point sets of the following types:

(1) $\left\{(x, y) \in M+F:(x-a)^{2}+y^{2}<r^{2}\right\}$ where $a \in U$ and $r>0$;

(2) $\{(z, y): u<y<v\}$ where $0<u<v<1$ and $z \in V$;

(3) $c+\left\{c_{i} \times I(0,1): i=m, m+1, \cdots\right\}^{*}$ where $c \in B$ and $m$ is a positive integer. (If $Q$ is a collection of point sets, then $Q^{*}$ denotes the union of all elements of Q.) 
M. E. Rudin proved that $S$ is not a subspace of a complete Moore space [6, Th. 8, p. 324].

The subspace $F$ is a complete metric space that is dense in $S$. Furthermore, $M+F$ is a metrizable open set and $B$ is a closed and isolated point set, hence $S$ is locally metrizable.

Suppose $T$ is a subspace of $S$. Let $B^{\prime}$ be the set of all points of $T \cdot B$ that are not limit points of $T \cdot(M+F)$. Then $B^{\prime}+T \cdot(M+F)$ is a metrizable dense subspace of $T$. Thus, every subspace of $S$ contains a dense metric subspace.

\section{BIBLIOGRAPHY}

1. O. H. Alzoobaee, Completion of Moore spaces, Ph. D. thesis, State University of Iowa City (1962).

2. B. Fitzpatrick, Jr., On dense subspaces of Moore spaces, (to appear).

3. R. L. Moore, Foundations of point set theory, rev. ed., Amer. Math. Soc. Colloq. Pub. 13, Amer. Math. Soc., Providence, 1962.

4. - A set of axioms for plane analysis situs, Fund. Math. 25 (1935), 13-28.

5. J. H. Roberts, A property related to completeness, Bull. Amer. Math. Soc. 17 (1932), 835-838.

6. M. E. (Estill) Rudin, Concerning abstract spaces, Duke Math. J. 17 (1950), 317-327.

7. J. N. Younglove, Concerning dense metric subspaces of certain non-metric spaces, Fund. Math. 48 (1959), 15-25.

Received February 20, 1965. This paper is a condensation of the author's doctoral dissertation which was directed by Ben Fitzpatrick, Jr. and submitted to the graduate faculty of Auburn University. The author wishes to express his appreciation to the National Science Foundation for their financial assistance.

Auburn University

Auburn, Alabama 



\section{PACIFIC JOURNAL OF MATHEMATICS}

H. SAMELSON

Stanford University Stanford, California

R. M. BLUMENTHAL

University of Washington

Seattle, Washington 98105

\section{EDITORS}

\author{
*J. DugundJI \\ University of Southern California \\ Los Angeles, California 90007 \\ RICHARD ARENS \\ University of California \\ Los Angeles, California 90024
}

\section{ASSOCIATE EDITORS}

E. F. BeCKENBACH

B. H. NEUMANN

F. WOLF

K. YosidA

\section{SUPPORTING INSTITUTIONS}

UNIVERSITY OF BRITISH COLUMBIA

CALIFORNIA INSTITUTE OF TECHNOLOGY

UNIVERSITY OF CALIFORNIA

MONTANA STATE UNIVERSITY

UNIVERSITY OF NEVADA

NEW MEXICO STATE UNIVERSITY

OREGON STATE UNIVERSITY

UNIVERSITY OF OREGON

OSAKA UNIVERSITY

UNIVERSITY OF SOUTHERN CALIFORNIA
STANFORD UNIVERSITY

UNIVERSITY OF TOKYO

UNIVERSITY OF UTAH

WASHINGTON STATE UNIVERSITY

UNIVERSITY OF WASHINGTON

AMERICAN MATHEMATICAL SOCIETY CHEVRON RESEARCH CORPORATION TRW SYSTEMS

NAVAL ORDNANCE TEST STATION 


\section{Pacific Journal of Mathematics}

Vol. 18, No. 1

March, 1966

Edward Joseph Barbeau, Semi-algebras that are lower semi-lattices ...... 1

Steven Fredrick Bauman, The Klein group as an automorphism group

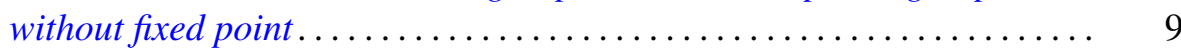

Homer Franklin Bechtell, Jr., Frattini subgroups and $\Phi$-central groups .... 15

Edward Kenneth Blum, A convergent gradient procedure in prehilbert

spaces ............................................

Edward Martin Bolger, The sum of two independent exponential-type random variables ...................................

David Wilson Bressler and A. P. Morse, Images of measurable sets .......

Dennison Robert Brown and J. G. LaTorre, A characterization of uniquely

divisible commutative semigroups........................

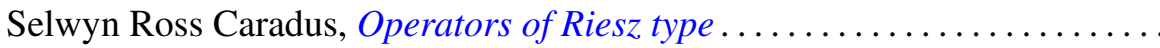

Jeffrey Davis and Isidore Isaac Hirschman, Jr., Toeplitz forms and ultraspherical polynomials ............................

Lorraine L. Foster, On the characteristic roots of the product of certain rational integral matrices of order two ......................

Alfred Gray and S. M. Shah, Asymptotic values of a holomorphic function

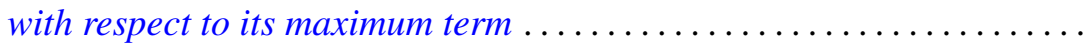

Sidney (Denny) L. Gulick, Commutativity and ideals in the biduals of

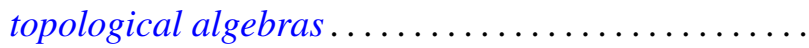

G. J. Kurowski, Further results in the theory of monodiffric functions

Lawrence S. Levy, Commutative rings whose homomorphic images are self-injective .

Calvin T. Long, On real numbers having normality of order $k$....

Bertram Mond, An inequality for operators in a Hilbert space. ...

John William Neuberger, The lack of self-adjointness in three-point boundary value problems ........................

C. A. Persinger, Subsets of $n$-books in $E^{3}$

Oscar S. Rothaus and John Griggs Thompson, A combinatorial problem in the symmetric group ............................... 175

Rodolfo DeSapio, Unknotting spheres via Smale .................. 179

James E. Shockley, On the functional equation

$$
F(m n) F((m, n))=F(m) F(n) f((m, n)) \ldots \ldots \ldots
$$

Kenneth Edward Whipple, Cauchy sequences in Moore spaces ... 